\title{
Responses to Visual Speech in Human Posterior Superior Temporal Gyrus Examined with iEEG Deconvolution
}

\author{
Brian A. Metzger, ${ }^{-}$John F. Magnotti, Zhengjia Wang, Elizabeth Nesbitt, Patrick J. Karas, Daniel Yoshor, \\ and ${ }^{\circledR}$ Michael S. Beauchamp \\ Department of Neurosurgery, Baylor College of Medicine, Houston, Texas 77030
}

\begin{abstract}
Experimentalists studying multisensory integration compare neural responses to multisensory stimuli with responses to the component modalities presented in isolation. This procedure is problematic for multisensory speech perception since audiovisual speech and auditory-only speech are easily intelligible but visual-only speech is not. To overcome this confound, we developed intracranial encephalography (iEEG) deconvolution. Individual stimuli always contained both auditory and visual speech, but jittering the onset asynchrony between modalities allowed for the time course of the unisensory responses and the interaction between them to be independently estimated. We applied this procedure to electrodes implanted in human epilepsy patients (both male and female) over the posterior superior temporal gyrus (pSTG), a brain area known to be important for speech perception. iEEG deconvolution revealed sustained positive responses to visual-only speech and larger, phasic responses to auditory-only speech. Confirming results from scalp EEG, responses to audiovisual speech were weaker than responses to auditory-only speech, demonstrating a subadditive multisensory neural computation. Leveraging the spatial resolution of iEEG, we extended these results to show that subadditivity is most pronounced in more posterior aspects of the pSTG. Across electrodes, subadditivity correlated with visual responsiveness, supporting a model in which visual speech enhances the efficiency of auditory speech processing in pSTG. The ability to separate neural processes may make iEEG deconvolution useful for studying a variety of complex cognitive and perceptual tasks.
\end{abstract}

Key words: audiovisual; human; intracranial; multisensory; speech perception; superior temporal gyrus

\section{Significance Statement}

Understanding speech is one of the most important human abilities. Speech perception uses information from both the auditory and visual modalities. It has been difficult to study neural responses to visual speech because visual-only speech is difficult or impossible to comprehend, unlike auditory-only and audiovisual speech. We used intracranial encephalography deconvolution to overcome this obstacle. We found that visual speech evokes a positive response in the human posterior superior temporal gyrus, enhancing the efficiency of auditory speech processing.

\section{Introduction}

When humans communicate face to face, auditory information from the talker's voice and visual information from the talker's mouth both provide clues about speech content. A critical brain area for multisensory speech perception is the posterior superior

Received Jan. 21, 2020; revised June 1, 2020; accepted June 2, 2020.

Author contributions: B.A.M., D.Y., and M.S.B. performed research; B.A.M., J.F.M., Z.W., E.N., P.J.K., and M.S.B. analyzed data; B.A.M. and M.S.B. wrote the first draft of the paper; B.A.M. and M.S.B. edited the paper; J.F.M. contributed unpublished reagents/analytic tools.

The authors declare no competing financial interests.

This work was supported by National Institutes of Health Grants R01NS065395, U01NS113339, R24MH117529, and R25NS070694.

Correspondence should be addressed to Michael S. Beauchamp at michael.beauchamp@bcm.edu. https://doi.org/10.1523/JNEUROSCI.0279-20.2020

Copyright @ 2020 Metzger et al.

This is an open-access article distributed under the terms of the Creative Commons Attribution License Creative Commons Attribution 4.0 International, which permits unrestricted use, distribution and reproduction in any medium provided that the original work is properly attributed. temporal gyrus and sulcus (pSTG), the location of human auditory association cortex (Moerel et al., 2014; Leaver and Rauschecker, 2016). The belt and parabelt areas in pSTG are selective for both the complex acoustic-phonetic features that comprise auditory speech (Belin et al., 2000; Formisano et al., 2008; Mesgarani et al., 2014) and the mouth movements that comprise visual speech (Beauchamp et al., 2004; Bernstein et al., 2011; Rhone et al., 2016; Ozker et al., 2017, 2018b; Zhu and Beauchamp, 2017; Rennig and Beauchamp, 2018; Beauchamp, 2019). However, the neural computations used by the pSTG to integrate auditory and visual speech features are poorly understood.

A widely used schema for understanding multisensory processing compares the amplitude of the responses to unisensory and multisensory stimuli (Stein and Stanford, 2008). If the responses to multisensory stimuli are greater than the sum of the responses to the component unisensory stimuli, the multisensory computation is termed "superadditive," suggesting the 
existence of facilitatory interactions between modalities. In contrast, if the multisensory responses are less than the sum of the unisensory responses, the computation is "subadditive," suggesting suppressive interactions between modalities. Multisensory responses equal to the sum of the unisensory responses are termed "additive," reflecting little or no interaction between modalities.

While this schema was codified in responses to simple auditory beep and visual flash stimuli in anesthetized animals (Stein and Stanford, 2008), it has also been applied to human brain responses to auditory and visual speech recorded with BOLD fMRI, scalp EEG, and intracranial EEG (iEEG) (Besle et al., 2004, 2008; van Wassenhove et al., 2005; Rhone et al., 2016; Karas et al., 2019). In these studies, responses to unisensory and multisensory speech were compared under the assumption that different sensory responses were the main driver of neural activity. However, perception of visual-only speech (lip-reading or speech-reading) places very different cognitive demands on humans, as it is both difficult and inaccurate: visual-only speech is largely unintelligible (Fig. 1A) (Peelle and Sommers, 2015). This lack of intelligibility could lead to decreased neural activity in language areas, which process semantics, increased activity in attentional control areas, or both, confounding assessment of the multisensory computation.

To circumvent this problem, we applied a novel experimental design inspired by the use of finite-impulse response functions to analyze event-related BOLD fMRI (Glover, 1999). In our design, all stimuli consisted of audiovisual speech, but the temporal onset asynchrony of the auditory and visual speech was varied (Fig. 1B). Then, deconvolution was applied to estimate the component unisensory responses and the multisensory computation (Fig. 1C). Presentation of audiovisual speech with a fixed asynchrony results in summed neural responses to the auditory and visual speech components that cannot be separated because of collinearity in the resulting system of linear equations. However, if the presentation of one component is delayed, the neural response for that component is also delayed. Repeating this process for different onset asynchronies allows the system of equations to be solved for the complete time course of the unisensory responses and the interaction between them (Fig. 1D).

If the value of the interaction term is near 0 , the measured audiovisual response will be equal to the sum of the auditory and visual responses, supporting an additive model of multisensory interactions during speech processing (Fig. 1E). If the interaction term is negative, the measured audiovisual response will be less than the sum of the estimated unisensory responses, indicating subadditivity (Fig. $1 F$ ). With a positive interaction term, the measured audiovisual response will be greater than the sum, indicating superadditive interactions between modalities (Fig. $1 G)$.

The key advantage of deconvolution is that it allows for the estimation of the responses to visual and auditory speech from measurements of the response to audiovisual speech presented with varying asynchrony. The temporal binding window for auditory and visual speech is on the order of hundreds of milliseconds (Grant and Seitz, 2000; Magnotti et al., 2013; Picton, 2013; Wallace and Stevenson, 2014) perhaps because of the large variability present in natural audiovisual speech (Chandrasekaran et al., 2009; Schwartz and Savariaux, 2014). This means that audiovisual speech with varying asynchrony is readily intelligible, avoiding the confounds introduced by comparing responses to intelligible audiovisual or auditory-only speech with responses to unintelligible visual-only speech.

\section{Materials and Methods}

\section{Human subjects}

All experiments were approved by the Committee for the Protection of Human Subjects at Baylor College of Medicine, and participants provided written informed consent. Participants consisted of 7 subjects (6 female, mean age 37 years, $6 \mathrm{~L}$ hemisphere) undergoing intracranial electrode grid placement for Phase 2 epilepsy monitoring. Electrode grids and strips were placed based on clinical criteria for epilepsy localization and resection guidance. All experiments were conducted in the epilepsy monitoring unit, and clinical monitoring continued unabated.

\section{Experimental design}

Visual stimuli were presented with an LCD monitor (Viewsonic VP150, $1024 \times 768$ pixels) placed on a stand located $57 \mathrm{~cm}$ in front of the subject's face. Auditory stimuli were presented through two speakers mounted on the wall behind and above the patient's head. Stimuli were presented using the Psychtoolbox extensions for MATLAB (Brainard, 1997; Pelli, 1997; Kleiner et al., 2007).

The stimuli consisted of audiovisual recordings of four different words ("back," "beach," "doubt," and "pail") recorded at $30 \mathrm{~Hz}$ (video) and $44.1 \mathrm{kHz}$ (audio) selected from the Hoosier Audiovisual Multitalker Database (Lachs and Hernandez, 1998; Conrey and Pisoni, 2004). Visual speech onset was defined as the time of the first video frame containing a visible mouth movement related to speech production. Auditory speech onset was defined as the first positive deflection in the auditory envelope corresponding to the beginning of the speech sound. Relative to the beginning of the recording, the visual onset/auditory onsets were as follows: "back," $500 \mathrm{~ms} / 631 \mathrm{~ms}$; "beach," $367 \mathrm{~ms} / 551 \mathrm{~ms}$; "doubt," $367 \mathrm{~ms} / 478 \mathrm{~ms}$; "pail," $433 \mathrm{~ms} / 562 \mathrm{~ms}$. This produced onset asynchronies of $131,184,111$, and $129 \mathrm{~ms}$, respectively. On average, the visual onset occurred $139 \mathrm{~ms}$ before the auditory onset.

There were three asynchrony conditions (Fig. 1B). The first condition consisted of unedited audiovisual recordings. The second condition consisted of auditory-advanced words for which the audio component was shifted forward in time by $300 \mathrm{~ms}$ in Adobe Premiere. The third condition consisted of visual-advanced words for which the video component was shifted forward in time by $300 \mathrm{~ms}$. This resulted in 12 total stimuli (4 stimulus exemplars $\times 3$ asynchrony conditions). Stimuli were presented in random order; and after each trial, participants responded via keypress whether they perceived the audiovisual speech as synchronous or asynchronous.

\section{iEEG data collection}

Neural signals were recorded with subdural grid and strip electrodes (2.3 $\mathrm{mm}$ exposed diameter platinum alloy discs embedded in flexible SILASTIC sheets; Ad-Tech) connected to a Cerebus data acquisition system (Blackrock Microsystems). A reversed intracranial electrode facing the skull was used as a reference for recording, and all signals were amplified, filtered (high-pass $0.3 \mathrm{~Hz}$ first-order Butterworth, low-pass $500 \mathrm{~Hz}$ fourth-order Butterworth), and digitized at $2000 \mathrm{~Hz}$. A photodiode was placed on the stimulus monitor to capture the exact time of visual stimulus onset. Both the photodiode output and the auditory output of the stimulus presentation computer were recorded by the data acquisition system to ensure precise synchronization between sensory stimulation and the evoked neural response.

Software tools and availability

All data analysis was conducted using the software tool RAVE (R Analysis and Visualization of intracranial Electroencephalography; freely available for Windows, Mac, and Linux platforms at https://openwetware.org/wiki/ RAVE).

\section{iEEG data analysis}

Preprocessing. Data were notch filtered $(60 \mathrm{~Hz}$, first and second harmonics) and converted into frequency and phase domains using a wavelet transform. The number of cycles of the wavelet was increased as a function of frequency, from three cycles at $2 \mathrm{~Hz}$ to 20 cycles at $200 \mathrm{~Hz}$, to optimize trade-off between temporal and frequency precision (Cohen, 2014). Data were downsampled to $100 \mathrm{~Hz}$ after the wavelet transform 
A
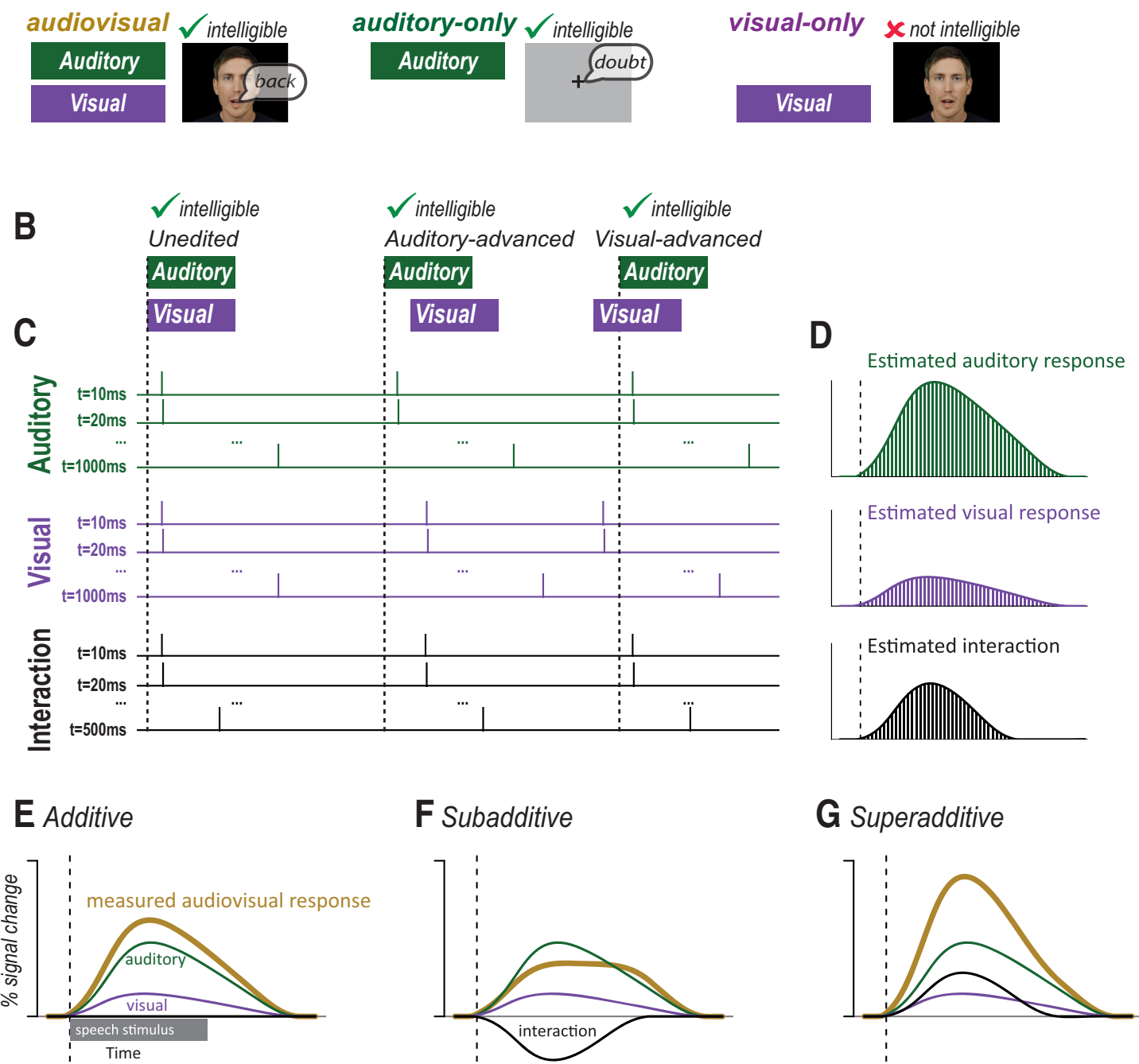

F Subadditive

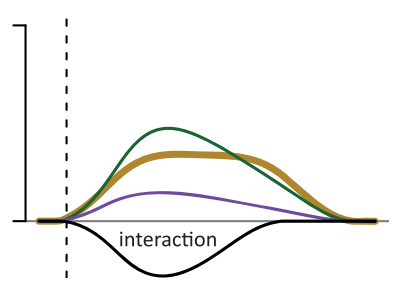

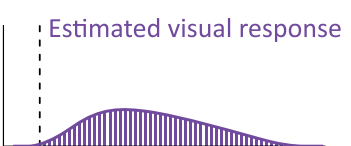

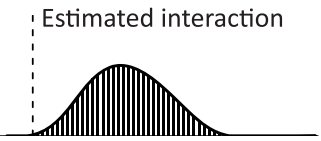

G Superadditive

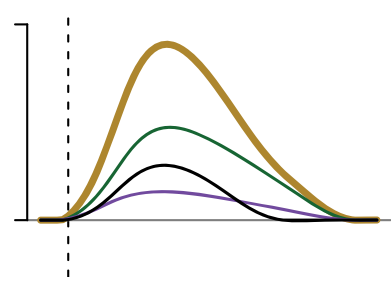

Figure 1. A, In many studies, audiovisual speech, auditory-only speech, and visual-only speech are presented. Comparing neural responses to these stimuli is confounded by the fact that audiovisual and auditory-only speech is intelligible whereas visual-only speech is not. $\boldsymbol{B}$, An alternative approach is to present audiovisual speech with varying asynchrony. Audiovisual speech can be unedited (synchronous), edited so that the auditory component of the speech is moved earlier (auditory-advanced), or edited so that the visual component of the speech is moved earlier (visual-advanced). All three types of speech are intelligible. C, Modifying the synchrony of the auditory and visual speech components allowed for the component responses to be estimated using deconvolution. The responses to auditory and visual speech were estimated with one predictor for each $10 \mathrm{~ms}$ time point of the response. Each predictor consists of a $\delta$ or tent function with amplitude of 1 at a single time point of the response and an amplitude of 0 at other time points (for simplicity, only the first, second, and last predictors are shown). Ellipsis indicates the remainder of the predictors. The sum of the predictors were fit to the measured neural response using a GLM to create an independent estimate of the auditory and visual responses. If auditory and visual speech begins at the same time, as shown for the unedited stimulus, the Aud $\mathrm{t}_{\mathrm{t}}=10 \mathrm{~ms}$ and Vis $\mathrm{s}_{\mathrm{t}}=10 \mathrm{~ms}$ regressors are identical, making it impossible to determine their relative amplitudes. If additional stimuli are presented for which the auditory speech and visual speech are temporally asynchronous, as shown for the auditory-advanced and visual-advanced stimuli, then the Aud $\mathrm{t}_{\mathrm{t}} 10 \mathrm{~ms}$ and Vis $\mathrm{t}_{\mathrm{t}}=10 \mathrm{~ms}$ regressors are offset. If many stimuli are presented with different asynchronies, the complete time courses of the auditory and visual responses can be estimated. The auditory and visual responses were modeled for $1 \mathrm{~s}$ after speech onset; the interaction response was modeled for $500 \mathrm{~ms}$ after speech onset. For complete design matrix, see Extended Data Figures 1-1 and 1-2. D. After the amplitude of each regressor was estimated by fitting to the measured neural response to all stimuli, the value of each time point regressor was plotted to show the time course of the component responses. Bars represent the amplitude of the regressor modeling each individual time point of the response: green represents auditory; purple represents visual; black represents interaction. $\boldsymbol{E}$, Gold curve indicates the measured response to audiovisual speech. If there are little or no interactions between modalities, the measured response will be equal to the sum of the estimated auditory (green) and visual (purple) responses. The interaction term will be 0 (black line along $x$ axis). $\boldsymbol{F}$, If the interactions between modalities are subadditive, the measured response will be less than the sum of the estimated auditory (green) and visual (purple) responses. The interaction term will be negative. $\mathbf{G}$, If the interactions between modalities are superadditive, the measured response will be less than the sum of the estimated auditory (green) and visual (purple) responses. The interaction term will be positive.

and then rereferenced to the average of all valid channels, determined by visual inspection. The continuous data were epoched into trials using the auditory speech onset of each stimulus as the reference $(t=0)$.

For each trial and frequency, the power data were transformed into percentage signal change from baseline, where baseline was set to the average power of the response from -1.0 to $-0.5 \mathrm{~s}$ before auditory speech onset. This time window consisted of the intertrial interval, during which participants were shown a dark gray screen with a white fixation point.
The percent signal change from this prestimulus baseline was then averaged over frequencies from 70 to $150 \mathrm{~Hz}$ to calculate the broadband high-frequency activity (BHA).

Electrode localization and selection. FreeSurfer (RRID:SCR_001847) (Dale et al., 1999b; Fischl et al., 1999a) was used to construct cortical surface models for each subject from their preoperative structural T1 MRI scans. Post-implantation CT brain scans, showing the location of the intracranial electrodes, were then aligned to the preoperative structural 


\section{A Electrode Locations}
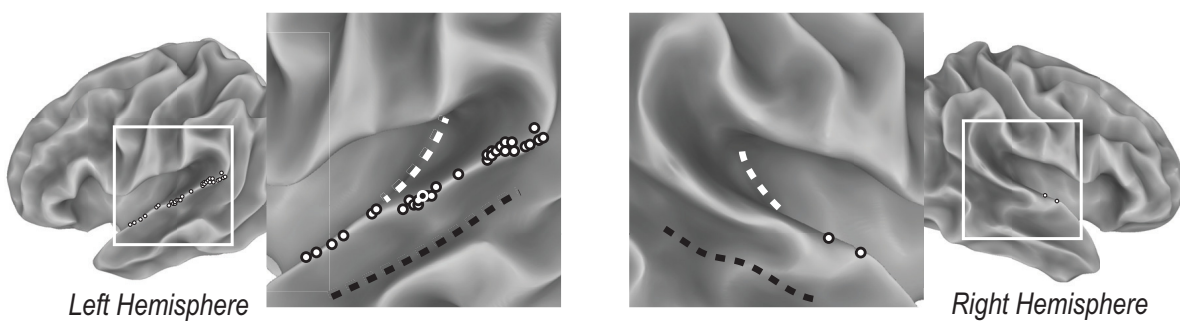

\section{B Additive model (single electrode)}

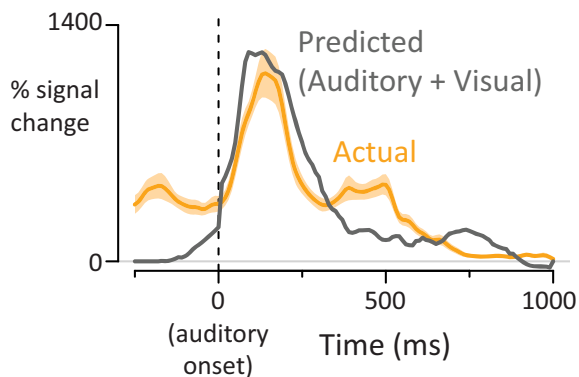

E Additive model (all electrodes)

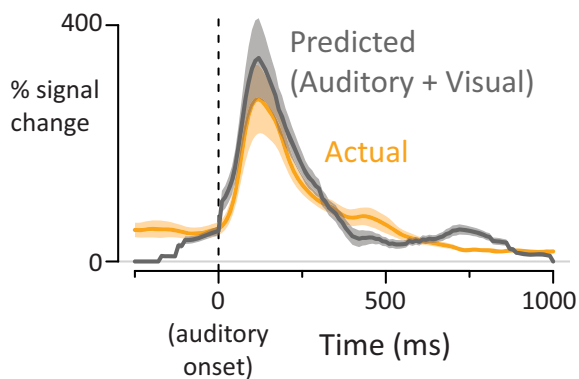

C Interaction model

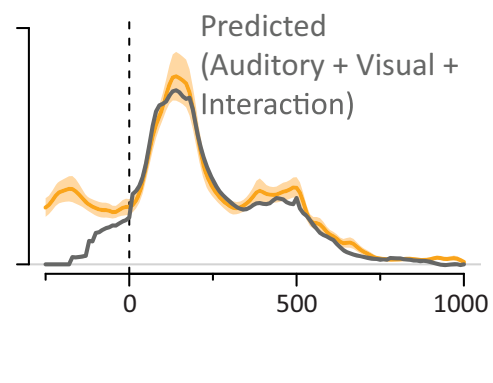

F Interaction model

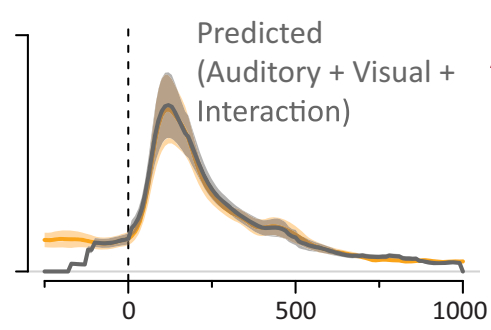

D Model error

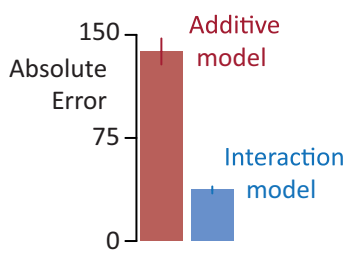

G Model error

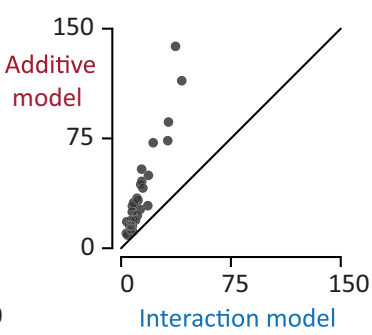

Figure 2. A, The location of left-hemisphere (left; $n=31$ ) and right-hemisphere (right; $n=2$ ) electrodes that met both an anatomic criterion (located on the posterior superior temporal gyrus) and a functional criterion (significant response to speech) displayed on a partially inflated cortical surface model. White dashed line indicates Heschl's gyrus. Black dashed line indicates the fundus of the superior temporal sulcus. Each electrode is shown as a white circle with a black outline, except for one electrode shown as a black circle with a white outline, corresponding to electrode YAR-22, responses shown in $\boldsymbol{B}-\boldsymbol{D}$. $\boldsymbol{B}$, Yellow line indicates the measured response of a single electrode (YAR-22) to audiovisual speech with auditory speech onset at time 0 and response in units of percent increase of BHA from prestimulus baseline. The mean \pm SEM (yellow shaded area) at each time point was calculated across trials. Gray line indicates the predicted time series from the additive model in which auditory and visual responses were summed. C, For this electrode, the predicted response (gray line) of the interaction model in which auditory, visual, and interaction estimates were summed. Actual response same as in $\boldsymbol{B}$. For measured and predicted responses for both models for all individual electrodes, see Extended Data Figure 2-1. $\boldsymbol{D}$, For this electrode, the error for each model was calculated by taking the absolute difference between the predicted and measured response at each time point and calculating the mean \pm SEM across time points. $E$, Yellow curve indicates the mean \pm SEM across electrodes of the response to audiovisual speech. For each electrode, an additive model was created in which auditory and visual responses were summed to generate a predicted audiovisual response. Gray curve indicates mean \pm SEM of the predicted response across electrodes. $\boldsymbol{F}$, For each electrode, an interaction model was created in which auditory, visual, and interaction responses were summed to generate a predicted response. Actual response same as in $\boldsymbol{E}$. G, Model error for the additive and interaction models plotted against each other, one symbol per electrode.

MRI brain using Analysis of Functional Neuroimaging (Cox, 1996). Electrode positions were marked manually using BioImage Suite 35 and projected to the nearest location on the cortical surface using iELVis (Groppe et al., 2017). SUMA in the Analysis of Functional Neuroimaging package was used to visualize cortical surface models with the overlaid electrodes, and positions were confirmed using intraoperative photographs of the electrode grids overlaid on the brain when available. For single-subject analysis, electrodes were visualized on that subject's cortical surface model. For group analysis, each participant's brain was aligned to the sulcal and gyral pattern of the Colin N27 brain (Holmes et al., 1998) using the FreeSurfer spherical template (Fischl et al., 1999b). Each surface was resampled to contain exactly 198,912 nodes, with the result that a given node index had the same anatomic location in any subject (Argall et al., 2006). Then, the node index closest to each electrode was determined in each subject, and the electrodes from all subjects displayed at the appropriate node in the N27 brain.
From a total of 786 electrodes implanted in 7 patients, $n=33$ electrodes were selected that were located in the PSTG and showed a significant neural response ( 31 left hemisphere, 2 right hemisphere; electrode locations shown in Fig. 2A). The pSTG was defined as the portion of STG located posteriorly to the central sulcus if the central sulcus continued in an inferior direction. A significant neural response was defined as $p<0.001$, Bonferroni-corrected BHA response to all speech words in the window from auditory stimulus onset to stimulus offset $(0-0.5 \mathrm{~s})$. Because the functional criterion ignored word type, the main comparisons of interest were independent of the functional criterion and hence unbiased.

Deconvolution. Inspired by analyses in which deconvolution was used to separate temporally overlapping responses in event-related fMRI designs (Glover, 1999), we used deconvolution to decompose the measured iEEG responses to audiovisual speech into responses to auditory and visual speech. In order to implement deconvolution in a GLM, a 
separate regressor was used to model every time point of the response (Fig. 1C). The regressors are independent, allowing neural responses to be modeled without any assumption as to their shape. In the resulting fitted models, the fit coefficient ( $\beta$-weight) for each regressor can be plotted (Fig. $1 D$ ), providing a best-fit estimate of the response over time to that stimulus (similar to a time-locked average).

The first model was an additive model in which auditory and visual speech evokes time-varying responses that sum at each time point to produce the measured response. To fit the additive model, we constructed two sets of regressors to estimate the time course of the response of each electrode to the auditory and visual components of speech. The time base of the regressors was the same as that of the data (with a value every $10 \mathrm{~ms}$ ), and the data window extended for $1 \mathrm{~s}$ from the onset of the given modality, resulting in 99 time points for each of the auditory and visual responses (the response at the time of stimulus onset was constrained to be 0 ). Each regressor consisted of a single-stick function (also known as a $\delta$ function or tent function) at the appropriate poststimulus time, and was 0 everywhere else, so that fitting the entire set of regressors modeled the time course of the response without any assumptions about its shape. As shown by the design matrix in Extended Data Figure 1-1, this resulted in a total of 198 different regressors, equivalent to 198 free parameters, one for each time point of the auditory and visual responses.

In event-related fMRI, a single-stimulus condition usually consists of presentation of multiple exemplars (e.g., a face, followed a few seconds later by a different face image, and so on); the deconvolved impulse response function represents the average response across all faces, although it is understood that individual stimuli evoke differing responses (Westfall et al., 2016). Our implementation of iEEG deconvolution is equivalent to this approach: the deconvolved visual, auditory, and interaction responses represent the average response across the four different word stimuli. An alternative approach would be to create separate deconvolution regressors for each word, but this would greatly increase the number of free parameters (to 198 parameters per word $\times 4$ words $=792$ free parameters).

Responses to the four different word stimuli were aligned so that the auditory onset of each word occurred at time 0 (this was necessary because the precise onset time of auditory speech within each unedited video clip varied slightly). The visual regressors were also shifted in time according to the timing of the visual speech in each clip. For instance, for the stimulus exemplar "doubt" in the visual-advanced condition, before fitting, the visual regressor was shifted to begin at $t=-411 \mathrm{~ms}$ and end at $t=589 \mathrm{~ms}$ to reflect the exact timing of the onset of visual speech for that exemplar/condition pair.

Deconvolution relies on variable asynchrony between the events to prevent collinearity (Fig. 1C). In rapid-event-related fMRI experiments, randomized stimulus schedules are used that introduce variable asynchrony between different trial types (Dale et al., 1999a). In our design, collinearity was addressed using the auditory-visual asynchrony in the 12 different trial types. In particular, the auditory-advanced and visualadvanced conditions had the least overlap between the auditory and visual regressors, reducing collinearity. For the additive model, ordinary least squares regression was used to determine auditory and visual coefficients for every time point in the auditory-advanced and visualadvanced conditions, and these fitted coefficients were used to predict the response to the unedited condition by summing the auditory and visual coefficients at each time point. To provide the best possible prediction, as during the fitting process, the visual coefficients were aligned based on the actual auditory-visual onset asynchrony.

The second deconvolution model included an interaction term (similar to that used in an ANOVA) in addition to the unisensory auditory and visual responses. Like the unisensory responses, the interaction term was modeled by creating a set of stick functions, with a value 1 at the appropriate poststimulus time point and 0 everywhere else. While the unisensory responses were modeled for $1 \mathrm{~s}$ after stimulus onset, the interaction could only be modeled for $500 \mathrm{~ms}$; this was the duration for which most trials had both auditory and visual speech present. The first time point of the interaction term occurred at auditory onset, and the final time point occurred at the offset of visual speech in the naturalhead-start condition, $500 \mathrm{~ms}$ later. The interaction time course was composed of 50 different regressors, corresponding to one sample every $10 \mathrm{~ms}$, and were generated by taking the product of the two unisensory regressors $(\mathrm{A} \times \mathrm{V})$ with the additional constraint that the interaction could not occur $>600 \mathrm{~ms}$ after visual onset. Ordinary least squares fitting was used to estimate the coefficients at each time point for the auditory, visual, and interaction regressors simultaneously, across all conditions. Then, the three coefficients at each time point were summed to generate a predicted response. Extended Data Figure 1-2 provides a representation of the entire design matrix of the interaction model.

The fitting procedure was repeated independently for every electrode. Stimulus was not included in the model, resulting in the simplifying assumption that all stimulus exemplars evoked the same amplitude of auditory and visual responses. However, the relative timing of the auditory and visual stimulus onsets of each word was used in the model, reducing the effect of condition and word to a single value, that of the relative asynchrony of auditory and visual speech. The fitted model was used to predict the response to each word (while the model contained only one coefficient for each regressor at each time point, it generated different predictions for each word and condition because of the audiovisual timing differences between them). To measure the goodness of fit, we compared the average (across words) predicted response with the actual average response in the unedited condition, from $100 \mathrm{~ms}$ before speech onset (to capture visual-only activity) to $1000 \mathrm{~ms}$ after speech onset. We calculated error by taking the absolute difference between the predicted and actual curves at each time point. Averaging error across time points provided a single measure of error for each electrode.

\section{Deconvolution model comparison}

Linear mixed-effects modeling was used to compare the fits of the additive model (with only auditory and visual terms) and the interaction model (with auditory, visual, and interaction terms) to the responses in each electrode (Schepers et al., 2014; Ozker et al., 2017, 2018a; Karas et al., 2019; Sjerps et al., 2019). The lme4 package was used for model construction (Bates et al., 2015), followed by $t$ tests with Satterthwaiteapproximated degrees of freedom (Kuznetsova et al., 2017). The dependent variable was model fit (absolute error). The fixed factor was model type (additive vs interaction, with additive used as the baseline). The random factors were participant and electrode nested within participant.

\section{Estimates of variance}

In order to visualize the variance of the measured response to audiovisual speech in a single electrode (Fig. $2 B, C$ ), the response at each $10 \mathrm{~ms}$ time point of the response was measured in each trial, and the mean \pm SEM at each time point across trials was calculated. The deconvolution models were fit to the data, producing a single predicted time course for the additive model and a different predicted time course for the interaction model. The average absolute error for each model was calculated by taking the absolute difference between the predicted and measured response at each time point and calculating the mean \pm SEM across time points (Fig. 2D).

To calculate the variance of the measured response to audiovisual speech across all electrodes, the mean \pm SEM at each time point across electrodes was calculated (Fig. $1 E, F$ ). The variance of the model fits was calculated the same way, by taking the average and SEM across electrodes at each time point (Fig. 1E,F). The variance of the estimated auditory, visual, and interactions responses was also calculated as the mean \pm SEM for each time point (regressor) across electrodes (see Fig. $3 A$ ).

\section{Spatial analysis}

For each electrode, an $x, y, z$ coordinate was assigned from the node closest to that electrode in a cortical surface model of the N27 brain in MNI space. The $y$ value of the standard space coordinate provided the electrode's location along the anterior-to-posterior axis of the pSTG (Ozker et al., 2017, 2018b). To measure the correspondence between electrode location and iEEG responses, we correlated the location of the 31 electrodes on the left pSTG with other values (there were only two electrodes over right $\mathrm{pSTG}$, too few to analyze). In some reporting conventions, more posterior anatomic locations are defined as more positive on the anterior-to-posterior axis; whereas in others, more posterior locations 
A Modelled responses

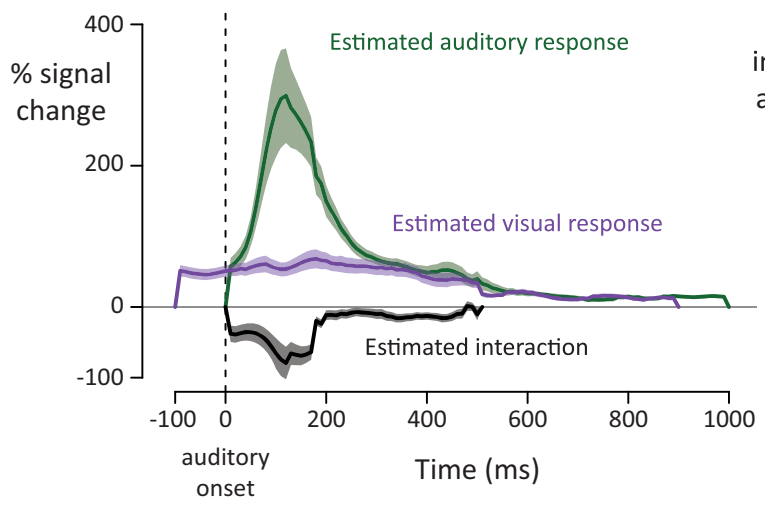

C Visual amplitude and anatomy

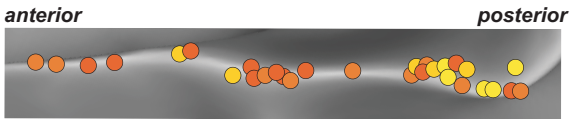

B

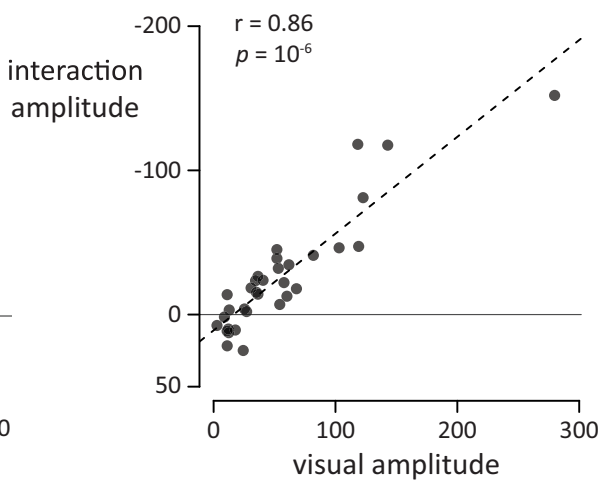

\section{Interaction amplitude and anatomy}
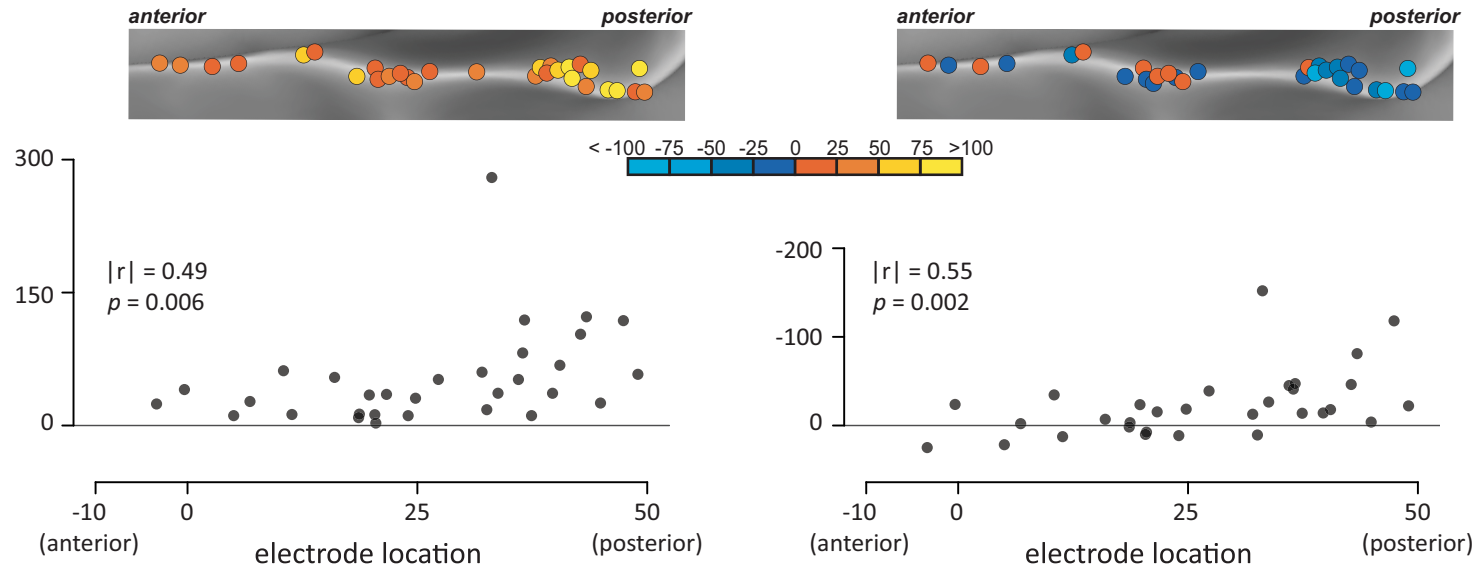

Figure 3. $\quad \boldsymbol{A}$, For each electrode, an interaction model was fit to generate modeled auditory (green), visual (purple), and interaction (black) time courses. Plots represent mean \pm SEM across all electrodes. For estimated responses for all individual electrodes, see Extended Data Figure 3-1. B, For each electrode, the visual response was plotted against the average interaction response, one symbol per electrode. C, All left hemisphere electrodes (rotated version of Fig. 2A, left) colored by their visual response amplitude. Bottom, Plot of anterior-to-posterior location in standard space against visual response amplitude. $\boldsymbol{D}$, Left hemisphere electrodes colored by their interaction amplitude. Bottom, Plot of the same data.

are defined as more negative. To avoid confusion, we report the absolute value of the spatial correlation values along with an explicit statement about the direction of the correlation (e.g., "greater visual response for more posterior electrodes").

Spearman rank correlations were used to minimize the influence of extreme values. The electrode locations were correlated with the visual response converted to a single value by averaging the deconvolution time point regressors over a $500 \mathrm{~ms}$ window beginning at the onset of the visual regressor. A single value for the interaction response was calculated by averaging the deconvolution regressors in a $500 \mathrm{~ms}$ window beginning at the onset of the deconvolution regressor.

To determine whether these spatial patterns were consistent across participants, we also fit linear mixed-effects models. Along with the fixed effect of electrode location, the models included random intercepts and random slopes for each participant. If the spatial pattern was driven by interparticipant variability, the random slope term will capture this variance and the fixed effect of spatial location will not be statistically significant $\left(\chi^{2}\right.$ test). To make this analysis commensurate with the Spearman correlation analysis, each variable was converted to a rank value before being entered into the model.

\section{Results}

We examined the response to audiovisual speech with varying asynchrony in 33 pSTG electrodes from 7 patients (electrode locations shown in Fig. 2A). One goal of the analysis was to determine whether interactions between neural responses to auditory and visual speech were additive, subadditive, or superadditive. The first step in making this determination was to compare two different deconvolution models: an additive model and an interaction model. Both models used as input the time course of the neural response to audiovisual words, measured as the average percent increase in the power of the broadband high-frequency electrical activity $(70-150 \mathrm{~Hz})$ relative to prestimulus baseline, with all responses time-locked to the onset of auditory speech. A superior fit of the additive model would indicate little or no interaction between modalities. A superior fit of the interaction model would indicate subadditivity (if the interaction was negative) or superadditivity (if the interaction was positive).

The additive model decomposed the measured response into two separate time courses, an auditory response and a visual response, under the null hypothesis of no interaction between the modalities. As shown for a single electrode in Figure $2 B$, the prediction of the additive model was a poor fit to the measured response (Fig. $2 B$ ) with a predicted peak response much greater than the actual peak response (1239\% vs $1114 \%$ increase from baseline).

The interaction model decomposed the measured response into three separate time courses: an auditory response, a visual response, and their interaction. The interaction model was a better fit to the measured response (Fig. 2C) with similar predicted versus actual peak values (1031\% vs $1114 \%$ ). 
To compare the goodness of fit of the two models, we measured the total error over the entire response to auditory speech ( -100 to $1000 \mathrm{~ms}$ after auditory speech onset). For this electrode, the total error for the additive model was more than twice the error of the interaction model (Fig. 2D; $145 \%$ vs $43 \%$ ).

Next, we fit the additive and interaction deconvolution models to all 33 pSTG electrodes. Averaged across electrodes, the additive model was a poor fit to the measured response with the predicted peak response greater that the actual peak (Fig. 2E; $344 \%$ vs $274 \%$ ). In contrast, the interaction model produced a better fit with similar predicted and actual peak responses (Fig. $2 F ; 282 \%$ vs $274 \%$ ). To show the results for all individual electrodes, we plotted the total error of both models against each other (Fig. 2G). All of the electrodes were above the line of identity, indicating more error for the additive model. To quantify the model difference across electrodes, a linear mixed-effects model with fixed factor of model type (additive vs interaction, with additive used as the baseline) and random factors of participant and electrode nested within participant was used to compare the models. There was a significant effect of model type, with a parameter estimate of $-22 \pm 4\left(t_{(32)}=-5.97, p=10^{-6}\right)$, demonstrating significantly better fit for the interaction model.

\section{Nature of the auditory, visual, and interaction responses}

Examining the modeled auditory, visual, and interaction terms allowed us to better understand the multisensory computations in pSTG (Fig. 3A). Averaged across electrodes, the estimated auditory BHA showed a rapid rise from auditory onset $(0 \mathrm{~ms})$ to a peak value of $299 \%$ at $120 \mathrm{~ms}$; the mean value of the auditory response in the first half-second of the response was $116 \%$. The interaction time series also showed a transient response, but of opposite polarity to the auditory response, with a peak deflection of $-79 \%$ at $130 \mathrm{~ms}$ and an average amplitude of $-26 \%$ in the first half-second. The visual response showed a different profile, characterized by a more sustained time course without clear peaks. The mean value was $55 \%$ in the first half-second of the visual response.

\section{Heterogeneity across electrodes}

There was substantial heterogeneity in the responses of different electrodes. While all electrodes showed a positive response to visual speech, the amplitude of the visual response (averaged of first $500 \mathrm{~ms}$ ) varied between electrodes, from $3 \%$ to $280 \%$. The amplitude of the interaction response also varied greatly between electrodes, from $-152 \%$ to $25 \%$; for 25 of 33 electrodes, the interaction value was negative, indicating a subadditive interaction (mean value $-26, V=79, p=10^{-4}$, from a nonparametric Wilcoxon test similar to a paired $t$ test). To determine whether the heterogeneity in visual and interaction amplitudes were related, we correlated the two values (all reported correlations are Spearman rank correlations to minimize the influence of extreme values). Electrodes with positive visual responses showed negative interaction terms, producing a strong negative correlation between the two quantities $\left(r_{(31)}=\right.$ $-0.86, p=10^{-6}$; Fig. 3B).

This high correlation could be the result of an uninteresting effect: more responsive electrodes might simply show larger auditory, visual, and interaction effects. To determine whether this was the case, we performed a partial correlation analysis that predicted the interaction response from the auditory and visual response, electrode by electrode. Taking changes in the auditory response magnitude into consideration, the visual response remained predictive of the interaction $\left(r_{(31)}=-0.81, p=10^{-7}\right)$. In contrast, with visual responses taken into consideration, the size of the auditory response did not predict the size of the interaction $\left(r_{(31)}=0.04\right.$,

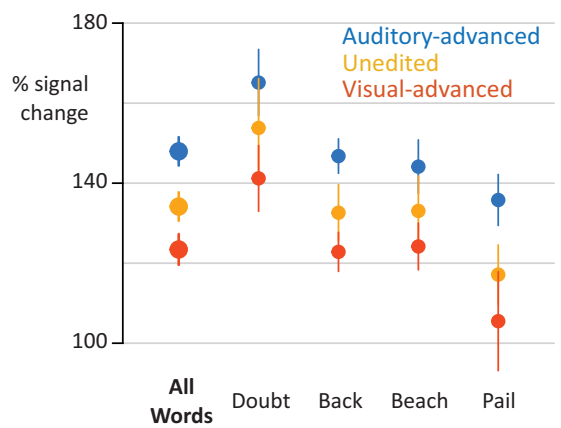

Figure 4. Responses to individual stimulus exemplars. y axis indicates percent increase in BHA relative to baseline. Points are colored by asynchrony. Error bars indicate within-subject SEM.(see Extended Data Fig. 1-1). For the additive model, auditory (green) and visual (purple) regressors were fit to auditory-advanced words (left column) and visual-advanced words (right column). Unisensory responses were modeled using 99 regressors, starting $10 \mathrm{~ms}$ after auditory onset, spaced at $10 \mathrm{~ms}$ intervals. Each regressor consisted of a single-tent or stick function (vertical colored line) that was 1 at the appropriate poststimulus onset time and 0 everywhere else (as shown in Fig. 10). For efficiency, all 99 regressors are shown collapsed onto a single time axis. The auditory regressors for the four different words ("back," "beach," "pail," and "doubt") were aligned to auditory speech onset (dashed gray line). The visual regressors for the four different words were adjusted to reflect the actual onset time of visual speech for each word.

$p=0.84)$. The results of the partial correlation analysis demonstrate that the amplitude of the visual response, but not the auditory response, predicted the size of the interaction.

\section{Anatomical differences in the response}

More posterior electrodes showed a greater visual response (Fig. $3 C$; spatial correlation between anterior-to-posterior electrode location and visual response; $\left.\left|r_{(29)}\right|=0.49, p=0.006\right)$ and more negative interaction values (Fig. $3 D ;\left|r_{(29)}\right|=0.55, p=0.002$ ). Linear mixed-effects models were constructed to estimate whether these spatial patterns were consistent across participants. The models found a significant relationship between electrode location and visual response $\left(\chi_{(1)}^{2}=7.2, p=0.007\right)$ and interaction size $\left(\chi_{(1)}^{2}=6.3, p=0.01\right)$.

As would be expected given that posterior electrodes had stronger interactions, posterior electrodes were poorly fit by the additive model (correlation between anterior-to-posterior location and additive model error minus interaction model error; $\left.\left|r_{(29)}\right|=0.39, p=0.03\right)$.

Across electrodes, the median time of peak interaction was $160 \mathrm{~ms}$. To determine whether this value changed along the pSTG, we estimated the time of peak interaction for each individual electrode. More posterior electrodes showed a shorter time-to-peak of the interaction $\left(\left|r_{(29)}\right|=0.41, p=0.02\right)$.

\section{Comparison of deconvolved responses with direct measurements}

Next, we sought to check the reasonableness of the estimated responses. Qualitatively, the deconvolved responses to auditory and visual speech measured in the present study appeared similar to the direct measurements of responses to auditory-only and visual-only speech in an earlier study (Karas et al., 2019). To quantify the similarity, we took advantage of the fact that 1 subject with five speech-responsive electrodes located over pSTG participated in both studies. We correlated the magnitude of the BHA power measured with deconvolution (data from the present study; stimuli consisting of audiovisual words with jitter) and BHA power measured directly (data from the earlier study; stimuli consisting of the auditory-only and visual-only words "drive" 
Table 1. Perceptual synchronous/asynchronous task performance ${ }^{a}$

\begin{tabular}{|c|c|c|c|c|c|c|c|c|c|c|}
\hline \multirow[b]{2}{*}{ Subject } & \multirow[b]{2}{*}{ N } & \multicolumn{3}{|l|}{ Unedited } & \multicolumn{3}{|c|}{ Auditory-advanced } & \multicolumn{3}{|c|}{ Visual-advanced } \\
\hline & & Synchronous & Asynchronous & Excluded & Synchronous & Asynchronous & Excluded & Synchronous & Asynchronous & Excluded \\
\hline YAI & 6 & 63 & 0 & 1 & 2 & 60 & 2 & 25 & 37 & 2 \\
\hline YAO & 6 & 59 & 0 & 1 & 0 & 59 & 1 & 57 & 1 & 2 \\
\hline YAQ & 3 & 58 & 0 & 2 & 5 & 52 & 3 & 48 & 10 & 2 \\
\hline YAR & 6 & 56 & 0 & 4 & 0 & 57 & 3 & 58 & 0 & 2 \\
\hline Total & & 396 & 1 & 15 & 77 & 316 & 19 & 335 & 62 & 15 \\
\hline Mean (\%) & & $96.1 \%$ & $0.2 \%$ & $3.6 \%$ & $18.7 \%$ & $76.7 \%$ & $4.6 \%$ & $81.3 \%$ & $15.0 \%$ & $3.6 \%$ \\
\hline
\end{tabular}

${ }^{a}$ For each subject, the number of electrodes and the number of trials for each asynchrony condition for which participants reported a perception of synchronous, asynchronous, or that were not analyzed (Excluded) because there was no response or the neural signal was noisy (measured voltage $>10$ SDs from the mean voltage).

and "last," not used in the current study). There was high overall correlation between the measured responses in the previous study and the deconvolution estimates produced here $\left(r^{2}=0.86\right.$ for $\mathrm{AV}, r^{2}=0.90$ for $\mathrm{A}, r^{2}=0.98$ for $\mathrm{V}$ ).

\section{Exemplar differences}

The present study presented four different audiovisual words at three different asynchronies. To quantify differences between stimulus conditions directly (without deconvolution), a single value for the response to each condition was calculated (mean BHA for first $500 \mathrm{~ms}$ after auditory onset). As shown in Figure 4, there were consistent differences across stimulus exemplars, with the largest response for auditory-advanced words, a smaller response to unedited words, and the smallest response to visualadvanced words. To quantify this observation, the response amplitudes entered into a linear mixed-effects model with a fixed factor of asynchrony (auditory-advanced, unedited, visual advanced) and random factors of stimulus exemplar, participant, and electrode nested within participant. The random effect of stimulus exemplar was modeled separately for each level of asynchrony, generating a random intercept and random slope for each exemplar across asynchrony. With this model, there was a significant effect of asynchrony $\left(\chi_{(2)}^{2}=17, p=10^{-4}\right)$, demonstrating that the effect of asynchrony was not driven by a particular stimulus exemplar.

\section{Perceptual reports}

After each trial, participants reported whether the auditory and visual speech was perceived as synchronous or asynchronous. Within each asynchrony condition, participants were strongly biased toward one percept or the other. Unedited words were perceived as synchronous on $96.1 \%$ of trials; visual-advanced words were rated as synchronous on $81.3 \%$ of the trials; auditoryadvanced words were rated as synchronous on only $18.7 \%$ of the trials (complete behavioral data in Table 1). Within conditions, stimuli were physically identical, but no single condition contained similar numbers of physically identical trials rated as perceptually different, rendering the data poorly suited for finding perceptually driven responses. Nevertheless, we compared the amplitude of the neural response within each condition to physically identical stimuli that received different perceptual ratings. There were no significant differences in pSTG response as a function of perceptual response for auditory-advanced words ( $141 \%$ for synchronous rating vs $149 \%$ for asynchronous rating; two-sample $t$ test $t_{(52)}=0.24, p=0.81$ ) or for visual-advanced words ( $122 \%$ for synchronous vs $97 \%$ for asynchronous; two- sample $t$ test $\left.t_{(52)}=0.22, p=0.83\right)$. There were too few unedited trials rated as asynchronous to perform the comparison.

\section{Discussion}

Using iEEG, we recorded neural responses to audiovisual speech in the human pSTG. While individual stimuli always contained both auditory and visual speech, jittering the onset asynchrony between auditory and visual speech allowed for the time course of the unisensory responses to be estimated using deconvolution. The response to visual speech began at the onset of the talker's face and showed a flat, sustained response profile. Auditory responses in pSTG were several-fold larger than the visual responses and showed a different response profile, with a transient rise and fall peaking at $130 \mathrm{~ms}$ after auditory speech onset. The interaction between auditory and visual speech was subadditive, with a weaker response to audiovisual speech than auditory speech alone, and the degree of subadditivity was correlated with the amplitude of the visual response. Multisensory interactions were strongest in more posterior sections of the pSTG.

\section{Utility of iEEG deconvolution}

Understanding responses to different sensory modalities in isolation is important for understanding the neural computations underlying multisensory integration. However, visual-only speech is an unnatural stimulus that is confusing or even aversive. While we can easily understand auditory-only speech (e.g., during a phone conversation), visual-only speech is not intelligible. This is a serious confound, as the lack of semantic information might dampen activity in areas of the language network important for semantic processing, or upregulate areas important for arousal and attention. Deconvolution allowed for the estimation of responses to visual-only speech while avoiding the confounds inherent in presenting it.

It will be important for future studies to investigate how audiovisual interactions differ between the single words used in the present study and the more ethologically relevant stimulus of continuous speech. While auditory-only and audiovisual continuous speech are engaging and easy to understand, participants often report giving up entirely when presented with continuous visual-only speech. By varying the asynchrony, iEEG deconvolution should allow visual and auditory speech components of continuous speech to be separated without this confound. For continuous speech, top-down factors, such as expectation and context, are also likely to play an important role in modulating pSTG responses (Heald and Nusbaum, 2014; Hickok and Poeppel, 2015; Tuennerhoff and Noppeney, 2016). 
The ability of iEEG deconvolution to separate neural responses to auditory and visual speech with only $600 \mathrm{~ms}$ of jitter between the two components is because of the high temporal resolution of iEEG. While our study focused on the high-frequency component of the iEEG signal, other studies have measured neural responses to audiovisual speech using event-related potentials (ERPs) derived from iEEG data (Besle et al., 2008), using MEG (Sohoglu and Davis, 2016) or EEG (Shahin et al., 2012). Deconvolution should be equally applicable to these other techniques since they share the temporal resolution necessary to record millisecond-by-millisecond neuronal responses. Two previous studies applied deconvolution to estimate responses to eye movements in iEEG (Golan et al., 2016) and EEG (Dandekar et al., 2012) datasets.

iEEG deconvolution should also be useful for measuring distinct neural processes underlying a variety of cognitive and perceptual tasks that consist of multiple components that are difficult to separate with subtraction logic, such as mental imagery (Sack et al., 2008) or the encoding, maintenance, and retrieval phases of working memory (Baddeley, 2012). Critically, deconvolution allows separation of neural responses to cognitive components that cannot be separated with the traditional Donders subtraction paradigm (Friston et al., 1996).

In $\mathrm{fMRI}$, the BOLD response to even a brief sensory stimulus lasts $\sim 15 \mathrm{~s}$. To increase experimental efficiency, in BOLD fMRI, rapid event-related designs are used that present stimuli with varying asynchrony on the time scale of seconds (Burock et al., 1998). Both the neural responses and the resulting hemodynamic signals are assumed to sum linearly (Glover, 1999; Henson, 2004). At the faster timescales of iEEG deconvolution, with stimuli jittered by hundreds of milliseconds rather than seconds, we found nonlinear interactions between auditory and visual speech responses: the response in most electrodes was better fit by a model that included an interaction term.

\section{Classification of multisensory computations}

A popular schema classifies multisensory neural responses as superadditive, additive, or subadditive by comparing the magnitudes of the responses to unisensory and multisensory stimuli (Stein and Meredith, 1993). To distinguish between additive and nonadditive multisensory responses, we fit an additive model and a model that included an interaction term, allowing for superadditivity or subadditivity. For 25 of 33 electrodes, the interaction term was negative, indicating subadditivity: the response to audiovisual speech was less than the response to auditory-only speech.

These results are consistent with studies recording brain responses from the scalp surface using EEG. In an important early study, Besle et al. (2004) studied responses to A, V, and AV syllables and found evidence for suppressive audiovisual integration. With similar stimuli, van Wassenhove et al. (2005) found that the N100/P200 ERP was weaker for audiovisual than auditory-only speech, consistent with the present results. Subadditivity for audiovisual speech has also been demonstrated for the N200 and N400 components of the speech ERP (Besle et al., 2004; Pilling, 2009; Ganesh et al., 2014; Paris et al., 2017). Applying the additivity classification scheme to BOLD fMRI data poses methodological difficulties (Beauchamp, 2005; Laurienti et al., 2005) and may explain early reports of superadditivity in pSTG (Calvert et al., 1999, 2000). Later BOLD fMRI studies typically report subadditivity (Wright et al., 2003; Nath and Beauchamp, 2011; Okada et al., 2013; but see Werner and Noppeney, 2010).

Recording with intracranial EEG in auditory cortex, Besle et al. (2008) found evidence for visually driven reductions in responses to auditory speech, although it is important to note that the ERPs used as a measure of neural activity by Besle et al. (2008) differ from the BHA measured in the present study. In general, BHA amplitude correlates with the rate of action potential firing by nearby neurons (Ray et al., 2008; Ray and Maunsell, 2011), although it may also include contributions from dendritic processing (Leszczynski et al., 2019) while ERPs are thought to reflect summed synaptic potentials (Buzsaki et al., 2012).

Besle et al. (2008) suggested that "preprocessing of the visual syllable would result in engaging less auditory resources from the auditory cortex," resulting in the observed reduction in auditory responses compared with visual responses. Building on this suggestion, in a recent paper, we suggested that this visual preprocessing could selectively inhibit populations of neurons response to auditory phonemes incompatible with the observed visual mouth shape (Karas et al., 2019). The model incorporates evidence that the pSTG contains neural populations that represent specific phonemes (Formisano et al., 2008; Mesgarani et al., 2014; Hamilton et al., 2018) and that visual information influences processing in auditory cortex (Calvert et al., 1997; Pekkola et al., 2005; Besle et al., 2008; Kayser et al., 2008; Zion Golumbic et al., 2013a,b; Rhone et al., 2016; Megevand et al., 2018; Ferraro et al., 2020). Reduced responses in pSTG to audiovisual speech may reflect more efficient processing, with less neural resources required to decode the speech. In turn, increased processing efficiency in pSTG could explain behavioral observations that audiovisual speech perception is faster, more accurate, and less effortful than auditory-only speech perception (Moradi et al., 2013). Support for a selective inhibition model is also provided by an elegant EEG experiment (Shahin et al., 2018). Using incongruent audiovisual speech pairings, visual speech modulated both the perception of an incongruent auditory syllable and rendered the evoked N100 component of the ERP similar to the congruent auditory condition. Suppressive interactions in higher-order cortex may be a general property of complex computations, as they have also been observed in other systems, including visuomotor integration in the frontal eye fields (Mirpour et al., 2018).

Although pSTG was classified by Brodmann as a single anatomic compartment (area 22), there is increasing evidence that anterior and posterior pSTG are functionally distinct (Ozker et al., 2017, 2018b; Hamilton et al., 2018). Even within parts of posterior pSTG that are visually responsive, there is evidence that the cortex is divided into subregions that respond to the visual mouth movements that make up visual speech and subregions that respond to viewed eye movements (Zhu and Beauchamp, 2017; Rennig and Beauchamp, 2018). The present study lends credence to the idea that there is substantial heterogeneity in this region, with multisensory interactions concentrated in the posterior portions of $\mathrm{pSTG}$.

\section{References}

Argall BD, Saad ZS, Beauchamp MS (2006) Simplified intersubject averaging on the cortical surface using SUMA. Hum Brain Mapp 27:14-27.

Baddeley A (2012) Working memory: theories, models, and controversies. Annu Rev Psychol 63:1-29.

Bates D, Mächler M, Bolker B, Walker S (2015) Fitting linear mixed-effects models using lme4. J Stat Softw 67:48.

Beauchamp MS (2005) Statistical criteria in FMRI studies of multisensory integration. Neuroinformatics 3:93-113.

Beauchamp MS (2019) Using multisensory integration to understand the human auditory cortex. In: Multisensory processes: the auditory perspective (Lee AK, Wallace MT, Coffin AB, Popper AN, Fay RR, eds), pp 161176. New York: Springer. 
Beauchamp MS, Argall BD, Bodurka J, Duyn JH, Martin A (2004) Unraveling multisensory integration: patchy organization within human STS multisensory cortex. Nat Neurosci 7:1190-1192.

Belin P, Zatorre RJ, Lafaille P, Ahad P, Pike B (2000) Voice-selective areas in human auditory cortex. Nature 403:309-312.

Bernstein LE, Jiang J, Pantazis D, Lu ZL, Joshi A (2011) Visual phonetic processing localized using speech and nonspeech face gestures in video and point-light displays. Hum Brain Mapp 32:1660-1676.

Besle J, Fort A, Delpuech C, Giard MH (2004) Bimodal speech: early suppressive visual effects in human auditory cortex. Eur J Neurosci 20:22252234.

Besle J, Fischer C, Bidet-Caulet A, Lecaignard F, Bertrand O, Giard MH (2008) Visual activation and audiovisual interactions in the auditory cortex during speech perception: intracranial recordings in humans. J Neurosci 28:14301-14310.

Brainard DH (1997) The Psychophysics Toolbox. Spat Vis 10:433-436.

Burock MA, Buckner RL, Woldorff MG, Rosen BR, Dale AM (1998) Randomized event-related experimental designs allow for extremely rapid presentation rates using functional MRI. Neuroreport 9:3735-3739.

Buzsaki G, Anastassiou CA, Koch C (2012) The origin of extracellular fields and currents: EEG, ECoG, LFP and spikes. Nat Rev Neurosci 13:407-420.

Calvert GA, Bullmore ET, Brammer MJ, Campbell R, Williams SC, McGuire PK, Woodruff PW, Iversen SD, David AS (1997) Activation of auditory cortex during silent lipreading. Science 276:593-596.

Calvert GA, Brammer MJ, Bullmore ET, Campbell R, Iversen SD, David AS (1999) Response amplification in sensory-specific cortices during crossmodal binding. Neuroreport 10:2619-2623.

Calvert GA, Campbell R, Brammer MJ (2000) Evidence from functional magnetic resonance imaging of crossmodal binding in the human heteromodal cortex. Curr Biol 10:649-657.

Chandrasekaran C, Trubanova A, Stillittano S, Caplier A, Ghazanfar AA (2009) The natural statistics of audiovisual speech. PLoS Comput Biol 5: e1000436.

Cohen MX (2014) Analyzing neural time series data: theory and practice. Cambridge, MA: Massachusetts Institute of Technology.

Conrey BL, Pisoni DB (2004) Detection of auditory-visual asynchrony in speech and nonspeech signals. Res Spoken Lang Process 26:71-94.

Cox RW (1996) AFNI: software for analysis and visualization of functional magnetic resonance neuroimages. Comput Biomed Res 29:162-173.

Dale AM, Greve DN, Burock MA (1999a) Optimal stimulus sequences for event-related fMRI. In: 5th International Conference on Functional Mapping of the Human Brain. Duesseldorf, Germany.

Dale AM, Fischl B, Sereno MI (1999b) Cortical surface-based analysis: I. Segmentation and surface reconstruction. Neuroimage 9:179-194.

Dandekar S, Privitera C, Carney T, Klein SA (2012) Neural saccadic response estimation during natural viewing. J Neurophysiol 107:1776-1790.

Ferraro S, Van Ackeren MJ, Mai R, Tassi L, Cardinale F, Nigri A, Bruzzone MG, D'Incerti L, Hartmann T, Weisz N, Collignon O (2020) Stereotactic electroencephalography in humans reveals multisensory signal in early visual and auditory cortices. Cortex 126:253-264.

Fischl B, Sereno MI, Dale AM (1999a) Cortical surface-based analysis. II: inflation, flattening, and a surface-based coordinate system. Neuroimage 9:195-207.

Fischl B, Sereno MI, Tootell RB, Dale AM (1999b) High-resolution intersubject averaging and a coordinate system for the cortical surface. Hum Brain Mapp 8:272-284.

Formisano E, De Martino F, Bonte M, Goebel R (2008) "Who" is saying "what"? Brain-based decoding of human voice and speech. Science 322:970-973.

Friston KJ, Price CJ, Fletcher P, Moore C, Frackowiak RS, Dolan RJ (1996) The trouble with cognitive subtraction. Neuroimage 4:97-104.

Ganesh AC, Berthommier F, Vilain C, Sato M, Schwartz JL (2014) A possible neurophysiological correlate of audiovisual binding and unbinding in speech perception. Front Psychol 5:1340.

Glover GH (1999) Deconvolution of impulse response in event-related BOLD fMRI. Neuroimage 9:416-429.

Golan T, Davidesco I, Meshulam M, Groppe DM, Megevand P, Yeagle EM, Goldfinger MS, Harel M, Melloni L, Schroeder CE, Deouell LY, Mehta AD, Malach R (2016) Human intracranial recordings link suppressed transients rather than 'filling-in' to perceptual continuity across blinks. Elife 5:e17243.
Grant KW, Seitz PF (2000) The use of visible speech cues for improving auditory detection of spoken sentences. J Acoust Soc Am 108:1197-1208.

Groppe DM, Bickel S, Dykstra AR, Wang X, Megevand P, Mercier MR, Lado FA, Mehta AD, Honey CJ (2017) iELVis: an open source MATLAB toolbox for localizing and visualizing human intracranial electrode data. J Neurosci Methods 281:40-48.

Hamilton LS, Edwards E, Chang EF (2018) A spatial map of onset and sustained responses to speech in the human superior temporal gyrus. Curr Biol 28:1860-1871.e1864.

Heald S, Nusbaum H (2014) Speech perception as an active cognitive process. Front Syst Neurosci 8:35

Henson R (2004) Analysis of fMRI timeseries: linear time-invariant models, event-related fMRI and optimal experimental design. In: Human Brain Function, Ed 2. Cambridge, MA: Academic Press.

Hickok G, Poeppel D (2015) Neural basis of speech perception. Handb Clin Neurol 129:149-160.

Holmes CJ, Hoge R, Collins L, Woods R, Toga AW, Evans AC (1998) Enhancement of MR images using registration for signal averaging. J Comput Assist Tomogr 22:324-333.

Karas PJ, Magnotti JF, Metzger BA, Zhu LL, Smith KB, Yoshor D, Beauchamp MS (2019) The visual speech head start improves perception and reduces superior temporal cortex responses to auditory speech. Elife 8:e48116.

Kayser C, Petkov CI, Logothetis NK (2008) Visual modulation of neurons in auditory cortex. Cereb Cortex 18:1560-1574.

Kleiner M, Brainard D, Pelli D, Ingling A, Murray R, Broussard C (2007) What's new in Psychtoolbox-3? Perception 36:1-16.

Kuznetsova A, Brockhoff PB, Christensen RH (2017) lmerTest package: tests in linear mixed effects models. J Stat Softw 82:1-26.

Lachs L, Hernandez LR (1998) Update: the Hoosier Audiovisual Multitalker Database. Res Spoken Lang Process 22:377-388.

Laurienti PJ, Perrault TJ, Stanford TR, Wallace MT, Stein BE (2005) On the use of superadditivity as a metric for characterizing multisensory integration in functional neuroimaging studies. Exp Brain Res 166:289-297.

Leaver AM, Rauschecker JP (2016) Functional topography of human auditory cortex. J Neurosci 36:1416-1428.

Leszczynski M, Barczak A, Kajikawa Y, Ulbert I, Falchier A, Tal I, Haegens S, Melloni L, Knight R, Schroeder C (2019) Dissociation of broadband high-frequency activity and neuronal firing in the neocortex. bioRxiv 531368. doi: 10.1101/531368.

Magnotti JF, Ma WJ, Beauchamp MS (2013) Causal inference of asynchronous audiovisual speech. Front Psychol 4:798.

Megevand P, Mercier MR, Groppe DM, Z, Golumbic E, Mesgarani N, Beauchamp MS, Schroeder CE, Mehta AD (2018) Phase resetting in human auditory cortex to visual speech. bioRxiv 405597. doi: 10.1101/ 405597.

Mesgarani N, Cheung C, Johnson K, Chang EF (2014) Phonetic feature encoding in human superior temporal gyrus. Science 343:1006-1010.

Mirpour K, Bolandnazar Z, Bisley JW (2018) Suppression of frontal eye field neuronal responses with maintained fixation. Proc Natl Acad Sci USA 115:804-809.

Moerel M, De Martino F, Formisano E (2014) An anatomical and functional topography of human auditory cortical areas. Front Neurosci 8:225.

Moradi S, Lidestam B, Ronnberg J (2013) Gated audiovisual speech identification in silence vs. noise: effects on time and accuracy. Front Psychol 4:359.

Nath AR, Beauchamp MS (2011) Dynamic changes in superior temporal sulcus connectivity during perception of noisy audiovisual speech. J Neurosci 31:1704-1714.

Okada K, Venezia JH, Matchin W, Saberi K, Hickok G (2013) An fMRI study of audiovisual speech perception reveals multisensory interactions in auditory cortex. PLoS One 8:e68959.

Ozker M, Schepers IM, Magnotti JF, Yoshor D, Beauchamp MS (2017) A double dissociation between anterior and posterior superior temporal gyrus for processing audiovisual speech demonstrated by electrocorticography. J Cogn Neurosci 29:1044-1060.

Ozker M, Yoshor D, Beauchamp MS (2018a) Frontal cortex selects representations of the talker's mouth to aid in speech perception. Elife 7:e30387.

Ozker M, Yoshor D, Beauchamp MS (2018b) Converging evidence from electrocorticography and BOLD fMRI for a sharp functional boundary in superior temporal gyrus related to multisensory speech processing. Front Hum Neurosci 12:141. 
Paris T, Kim J, Davis C (2017) Visual form predictions facilitate auditory processing at the N1. Neuroscience 343:157-164.

Peelle JE, Sommers MS (2015) Prediction and constraint in audiovisual speech perception. Cortex 68:169-181.

Pekkola J, Ojanen V, Autti T, Jaaskelainen IP, Mottonen R, Tarkiainen A, Sams M (2005) Primary auditory cortex activation by visual speech: an fMRI study at 3 T. Neuroreport 16:125-128.

Pelli DG (1997) The VideoToolbox software for visual psychophysics: transforming numbers into movies. Spat Vis 10:437-442.

Picton T (2013) Hearing in time: evoked potential studies of temporal processing. Ear Hear 34:385-401.

Pilling M (2009) Auditory event-related potentials (ERPs) in audiovisual speech perception. J Speech Lang Hear Res 52:1073-1081.

Ray S, Maunsell JH (2011) Different origins of gamma rhythm and highgamma activity in macaque visual cortex. PLoS Biol 9:e1000610.

Ray S, Crone NE, Niebur E, Franaszczuk PJ, Hsiao SS (2008) Neural correlates of high-gamma oscillations $(60-200 \mathrm{~Hz})$ in macaque local field potentials and their potential implications in electrocorticography. J Neurosci 28:11526-11536

Rennig J, Beauchamp MS (2018) Free viewing of talking faces reveals mouth and eye preferring regions of the human superior temporal sulcus. Neuroimage 183:25-36.

Rhone AE, Nourski KV, Oya H, Kawasaki H, Howard MA, McMurray B (2016) Can you hear me yet? An intracranial investigation of speech and non-speech audiovisual interactions in human cortex. Lang Cogn Neurosci 31:284-302.

Sack AT, Jacobs C, De Martino F, Staeren N, Goebel R, Formisano E (2008) Dynamic premotor-to-parietal interactions during spatial imagery. J Neurosci 28:8417-8429.

Schepers IM, Yoshor D, Beauchamp MS (2014) Electrocorticography reveals enhanced visual cortex responses to visual speech Cereb Cortex 25:41034110 .

Schwartz JL, Savariaux C (2014) No, there is no $150 \mathrm{~ms}$ lead of visual speech on auditory speech, but a range of audiovisual asynchronies varying from small audio lead to large audio lag. PLoS Comput Biol 10:e1003743.

Shahin AJ, Kerlin JR, Bhat J, Miller LM (2012) Neural restoration of degraded audiovisual speech. Neuroimage 60:530-538.
Shahin AJ, Backer KC, Rosenblum LD, Kerlin JR (2018) Neural mechanisms underlying cross-modal phonetic encoding. J Neurosci 38:1835-1849.

Sjerps MJ, Fox NP, Johnson K, Chang EF (2019) Speaker-normalized sound representations in the human auditory cortex. Nat Commun 10:2465.

Sohoglu E, Davis MH (2016) Perceptual learning of degraded speech by minimizing prediction error. Proc Natl Acad Sci USA 113:1747-1756.

Stein BE, Meredith MA (1993) The merging of the senses. Cambridge, MA: Massachusetts Institute of Technology.

Stein BE, Stanford TR (2008) Multisensory integration: current issues from the perspective of the single neuron. Nat Rev Neurosci 9:255-266.

Tuennerhoff J, Noppeney U (2016) When sentences live up to your expectations. Neuroimage 124:641-653.

van Wassenhove V, Grant KW, Poeppel D (2005) Visual speech speeds up the neural processing of auditory speech. Proc Natl Acad Sci USA 102:1181-1186

Wallace MT, Stevenson RA (2014) The construct of the multisensory temporal binding window and its dysregulation in developmental disabilities. Neuropsychologia 64:105-123.

Werner S, Noppeney U (2010) Superadditive responses in superior temporal sulcus predict audiovisual benefits in object categorization. Cereb Cortex 20:1829-1842.

Westfall J, Nichols TE, Yarkoni T (2016) Fixing the stimulus-as-fixed-effect fallacy in task fMRI. Wellcome Open Res 1:23.

Wright TM, Pelphrey KA, Allison T, McKeown MJ, McCarthy G (2003) Polysensory interactions along lateral temporal regions evoked by audiovisual speech. Cereb Cortex 13:1034-1043.

Zhu LL, Beauchamp MS (2017) Mouth and voice: a relationship between visual and auditory preference in the human superior temporal sulcus. J Neurosci 37:2697-2708.

Zion Golumbic E, Cogan GB, Schroeder CE, Poeppel D (2013a) Visual input enhances selective speech envelope tracking in auditory cortex at a "cocktail party." J Neurosci 33:1417-1426.

Zion Golumbic EM, Ding N, Bickel S, Lakatos P, Schevon CA, McKhann GM, Goodman RR, Emerson R, Mehta AD, Simon JZ, Poeppel D, Schroeder CE (2013b) Mechanisms underlying selective neuronal tracking of attended speech at a "cocktail party." Neuron 77:980-991. 